\title{
DIVERGENCE OF CONTINUED FRACTIONS RELATED TO HYPERGEOMETRIC SERIES
}

\author{
LISA LORENTZEN
}

\begin{abstract}
Let $K\left(a_{n} / b_{n}\right)$ be a limit periodic continued fraction of elliptic type; i.e., $a_{n} \rightarrow a$ and $b_{n} \rightarrow b$, where $a /(b+w)$ is an elliptic linear fractional transformation of $w$. We show that if $\sum\left|a_{n}-a\right|<\infty$ and $\sum\left|b_{n}-b\right|<\infty$, then $K\left(a_{n} / b_{n}\right)$ diverges. This generalizes the well-known Stern-Stolz Theorem. The Gauss continued fraction (related to hypergeometric functions) is used as an example. We also give an example where $a_{n}-a=\mathscr{O}\left(n^{-1}\right)$ and $b_{n}=b=1$ and $K\left(a_{n} / b_{n}\right)$ converges. The divergence result is also generalized further.
\end{abstract}

\section{INTRODUCTION}

A continued fraction

$$
\begin{aligned}
K\left(a_{n} / b_{n}\right) & =\mathrm{K} \frac{a_{n}}{b_{n}}={\underset{K}{n=1}}_{a_{1}}^{\infty} \frac{a_{n}}{b_{n}}=\frac{a_{1}}{b_{1}}+\frac{a_{2}}{b_{2}}+\frac{a_{3}}{b_{3}}+\cdots \\
& =\frac{a_{2}}{b_{1}+\frac{a_{3}}{b_{2}+\frac{a_{3}+\cdot}{b_{3}+\cdot}}}
\end{aligned}
$$

where $a_{n} \in \mathbf{C} \backslash\{0\}$ and $b_{n} \in \mathbf{C}$ for all $n$, is said to converge if its sequence of (classical) approximants

$$
f_{k}={\underset{K}{n=1}}_{k}^{k} \frac{a_{n}}{b_{n}}=\frac{a_{1}}{b_{1}}+\frac{a_{2}}{b_{2}}+\cdots+\frac{a_{k}}{b_{k}} \text { for } k=1,2,3, \ldots
$$

converges in $\hat{\mathbf{C}}=\mathbf{C} \cup\{\infty\}$. Its value is then $f=\lim f_{k}$. We say that $K\left(a_{n} / b_{n}\right)$ diverges if this limit does not exist in $\hat{\mathbf{C}}$. We adopt the convention that a continued fraction $K\left(a_{n} / b_{n}\right)$ has all $a_{n} \neq 0$ by definition.

The numerators $\left\{A_{n}\right\}_{n=-1}^{\infty}$ and denominators $\left\{B_{n}\right\}_{n=-1}^{\infty}$ of $K\left(a_{n} / b_{n}\right)$ are the solutions $\left\{X_{n}\right\}$ of the linear recurrence relation

$$
X_{n}=b_{n} X_{n-1}+a_{n} X_{n-2} \text { for } n=1,2,3, \ldots
$$

with initial values $B_{-1}=0, B_{0}=1, A_{-1}=1, A_{0}=0$. With this notation, the (modified) approximants $S_{n}(w)$ of $K\left(a_{n} / b_{n}\right)$ can be written

$$
S_{n}(w)=\frac{a_{1}}{b_{1}}+\frac{a_{2}}{b_{2}}+\cdots+\frac{a_{n}}{b_{n}+w}=\frac{A_{n}+A_{n-1} w}{B_{n}+B_{n-1} w} \text { for } n=1,2,3, \ldots
$$

Received by the editor February 14, 1992.

1991 Mathematics Subject Classification. Primary 40A15; Secondary 33C05.

Key words and phrases. Hypergeometric series, divergence of continued fractions.

The author has changed her name from Lisa Jacobsen. 
In particular, $f_{k}=S_{k}(0)=A_{k} / B_{k}$. We also have the well-known determinant formula

$$
A_{n} B_{n-1}-B_{n} A_{n-1}=(-1)^{n+1} \prod_{k=1}^{n} a_{k} .
$$

It is also useful to write $A_{n}^{(k)}$ and $B_{n}^{(k)}$ for the numerators and denominators of the $k$ th tail of $K\left(a_{n} / b_{n}\right)$, which is the continued fraction

$$
\frac{a_{k+1}}{b_{k+1}}+\frac{a_{k+2}}{b_{k+2}}+\frac{a_{k+3}}{b_{k+3}}+\cdots, \quad k \in \mathbf{N}_{0} \text {. }
$$

( $\mathbf{N}$ is the set of positive integers, and $\mathbf{N}_{0}=\mathbf{N} \cup\{0\}$.) Clearly, $A_{n}^{(0)}=A_{n}$ and $B_{n}^{(0)}=B_{n}$.

The aim of this paper is to find criteria for divergence of continued fractions. It is important to study divergence in order to learn more about convergence. This was realized already in 1860 when Stern [13] published the result that $K\left(1 / b_{n}\right)$ diverges if

$$
\sum\left|b_{n}\right|<\infty \text {. }
$$

(However, its even approximants $\left\{f_{2 n}\right\}$ and its odd approximants $\left\{f_{2 n+1}\right\}$ both converge to finite values.) This was also known to Stolz [14], and the result is called the Stern-Stolz Theorem. Later, Van Vleck [16] published his stunning result that if $\left|\arg \left(b_{n}\right)\right|<\pi / 2-\varepsilon$ for all $n$ for some $\varepsilon>0$, then $K\left(1 / b_{n}\right)$ diverges if and only if $(0.7)$ holds. So, in this sense, $(0.7)$ is an optimal criterion.

We shall generalize the Stern-Stolz Theorem in the next section. The proofs are given in $\S 2$, and $\S 3$ contains a further generalization.

\section{THE LIMIT PERIODIC CASE}

Let $K(a / b)$ be a periodic continued fraction of elliptic type; i.e., $a \in \mathbf{C} \backslash\{0\}$, $b \in \mathbf{C}$, and

$$
s(w)=\frac{a}{b+w}
$$

is an elliptic linear fractional transformation with $s(w)$ having two distinct fixed points $x$ and $y$ such that $|x|=|y|$. Actually, if $x$ is a fixed point for $s$, then $y=-(b+x)$ is its second fixed point. Hence $s$ is elliptic if and only if

$$
x \neq-(b+x) \text { and }|x|=|b+x| .
$$

It is then trivial to see that $K(a / b)$ diverges. (See, for instance, [8, p.47].) We want to study limit periodic continued fractions $K\left(a_{n} / b_{n}\right)$ of elliptic type; i.e. $a_{n} \rightarrow a$ and $b_{n} \rightarrow b$ where $a$ and $b$ are as above, and we shall study their tail sequences $\left\{t_{n}\right\}_{n=0}^{\infty}$; i.e., $t_{n} \in \hat{\mathbf{C}}$ and

$$
t_{n-1}=s_{n}\left(t_{n}\right)=\frac{a_{n}}{b_{n}+t_{n}} \text { for } n=1,2,3, \ldots .
$$

Theorem 1.1. Let $K\left(a_{n} / b_{n}\right)$ be limit periodic of elliptic type with

$$
\sum_{n=1}^{\infty}\left|a_{n}-a\right|<\infty \quad \text { and } \quad \sum_{n=1}^{\infty}\left|b_{n}-b\right|<\infty
$$


Then

A. $K\left(a_{n} / b_{n}\right)$ diverges.

B. Its (modified) approximants

$$
S_{n}(x)=\frac{a_{1}}{b_{1}}+\frac{a_{2}}{b_{2}}+\cdots+\frac{a_{n}}{b_{n}+x}=s_{1} \circ s_{2} \circ \cdots \circ s_{n}(x)
$$

converge to a limit $t_{0} \in \hat{\mathbf{C}}$ as $n \rightarrow \infty$, if $x$ is a fixed point of (1.1).

C. Its tail sequence $\left\{t_{n}\right\}$, with $t_{0}=\lim S_{n}(x)$, converges to $x$.

Remarks. 1. Part C implies that $\lim S_{n}(x) \neq \lim S_{n}(-b-x)$ in part B.

2. In part $B$ we actually prove the stronger result that

$$
\frac{A_{n}+A_{n-1} x}{(b+x)^{n}} \text { and } \frac{B_{n}+B_{n-1} x}{(b+x)^{n}}
$$

both converge separately to finite values which are not both equal to zero.

3. The way in which $K\left(a_{n} / b_{n}\right)$ diverges depends on the ratio $(b+x) /(-x)=$ $e^{i \theta}$. From the proof of Theorem 1.1 it follows that if $\left\{e^{i n \theta}\right\}_{n=0}^{\infty}$ is $k$-periodic (i.e., $\theta / \pi$ is a rational number), then $\left\{S_{n}(0)\right\}$ is limit $k$-periodic, and if $\theta / \pi$ is nonrational, then $\left\{S_{n}(0)\right\}$ has infinitely many limit points. For $a=1, b=0$ we have $\theta / \pi=-1$, and thus $\left\{S_{n}(0)\right\}$ is limit 2-periodic, just as we know from the Stern-Stolz Theorem.

4. Theorem 1.1 generalizes a result from [4].

5. Theorem 1.1 generalizes the Stern-Stolz Theorem since (1.1) is elliptic if $b=0$. (See (1.2).) Actually, we get slightly more, also in the Stern-Stolz situation, i.e., if $K\left(1 / b_{n}\right)$ diverges by (0.7). Then the two fixed points of $s$ are $x=1$ and $x=-1$, so that the limits of $S_{n}(1)$ and of $S_{n}(-1)$ both exist (and are distinct), and the tail sequence $\left\{t_{n}\right\}$ with $t_{0}=\lim S_{n}(1)$, converges to 1 , the one with $t_{0}=\lim S_{n}(-1)$ converges to -1 .

6. Theorem 1.1 really expresses the idea that if $K\left(a_{n} / b_{n}\right)$ is "near enough" to a given continued fraction $K(a / b)$, then $K\left(a_{n} / b_{n}\right)$ "behaves almost like" $K(a / b)$. This is an old and fruitful idea. See, for instance, [11, §19], [5], [15]. However, the emphasis has normally been on convergence rather than divergence. Still, it is possible also to derive Theorem 1.1A from [3, Theorem $2 \mathrm{~b}$ ] by a simple argument.

Let us see what consequences Theorem 1.1 has in a particular example: The Gauss continued fraction $1+K\left(a_{n} z / 1\right)$, where

$$
a_{2 n}=-\frac{(b+n)(c-a+n)}{(c+2 n-1)(c+2 n)}, \quad a_{2 n+1}=-\frac{(a+n)(c-b+n)}{(c+2 n)(c+2 n+1)},
$$

(see for instance [8, p.199]) converges to the ratio

$$
f(z)=\frac{{ }_{2} F_{1}(a, b ; c ; z)}{{ }_{2} F_{1}(a, b+1 ; c+1 ; z)}
$$

of hypergeometric functions in the cut plane $\{z \in \mathbf{C} ;|\arg (1-z)|<\pi\}$. But what happens on the cut $z>1$ ? A natural thought is that there the continued fraction diverges. And indeed, by means of Theorem 1.1A we can prove that this is so. 
Theorem 1.2. Let $1+K\left(a_{n} z / 1\right)$ be given by (1.7), where $a, b$, and $c$ are complex numbers chosen such that all $a_{n}$ are well-defined numbers in $\mathbf{C} \backslash\{0\}$. Then $1+K\left(a_{n} z / 1\right)$ diverges if either

A. $z>1$

or

B. $z=1$ and $c-a-b=$ it for $a t \in \mathbf{R} \backslash\{0\}$.

(The Gauss fraction converges for $z=1$ if $c=a+b$ or if $\operatorname{Re}(c-a-b) \neq 0$.)

Not every limit periodic continued fraction of elliptic type diverges. This is evident from Van Vleck's Theorem, quoted in the introduction. For instance, $K\left(1 / b_{n}\right)$ converges if all $0<b_{n} \rightarrow 0$ with $\sum b_{n}=\infty$. It was also proved by Gill [2] to be true in cases outside the Stern-Stolz situation, but no examples were given. The following theorem provides such an example:

Theorem 1.3. The continued fraction $K\left(a_{n} / 1\right)$, where

$$
a_{n}=-\left(\frac{1}{4}+t^{2}\right)+r \frac{2 i t n+\frac{1}{2}+i t+r}{n(n+1)} \text { for } n=1,2,3, \ldots
$$

with $r \in \mathbf{C}$ and $t \in \mathbf{R} \backslash\{0\}$, converges in $\hat{\mathbf{C}}$ if $\operatorname{Re}(r)<-\left(\frac{1}{4}+t^{2}\right)$.

We have implicitly assumed that $t$ and $r$ are chosen such that all $a_{n} \neq 0$.

Expression (1.9) may seem somewhat strange. But $K\left(a_{n} / 1\right)$ is chosen such that

$$
a_{n}=t_{n-1}\left(1+t_{n}\right), \quad t_{n-1}=-\frac{1}{2}+i t+\frac{r}{n} \text { for } n=1,2,3, \ldots ;
$$

i.e., $K\left(a_{n} / 1\right)$ is limit periodic of elliptic type, and $\left\{t_{n}\right\}$ is a tail sequence for $K\left(a_{n} / 1\right)$, in fact a tail sequence converging to $x=-\frac{1}{2}+i t$.

\section{PROOFS FOR THE LIMIT PERIODIC CASE}

We shall use the following lemmas to prove Theorem 1.1 .

Lemma 2.1. Let $\left\{t_{n}\right\}$ be a tail sequence for $K\left(a_{n} / b_{n}\right)$ such that all $t_{n} \neq \infty$. Then

$$
B_{n}=\sum_{k=0}^{n}\left(\prod_{m=1}^{k}\left(b_{m}+t_{m}\right) \prod_{m=k+1}^{n}\left(-t_{m}\right)\right) \text { for } n=0,1,2, \ldots
$$

Note that we also have all $t_{n} \neq 0$ and all $\left(b_{n}+t_{n}\right) \neq 0$ when all $t_{n} \neq \infty$. This follows from (1.3). The proof of Lemma 2.1 is by induction, using the recurrence relation $(0.3)$ and the fact that

$$
a_{n}=t_{n-1}\left(b_{n}+t_{n}\right) \quad \text { for } n=1,2,3, \ldots
$$

(See (1.3).) The formula (2.1) can essentially be found in [7].

Lemma 2.2. Let $\left\{B_{n}\right\}$ and $\left\{\tilde{B}_{n}\right\}$ be the denominators of $K\left(a_{n} / b_{n}\right)$ and $K\left(\tilde{a}_{n} / \tilde{b}_{n}\right)$, respectively. Then

$$
B_{n}=\tilde{B}_{n}+\sum_{k=1}^{n}\left(\left(b_{k}-\tilde{b}_{k}\right) \tilde{B}_{n-k}^{(k)}+\left(a_{k+1}-\tilde{a}_{k+1}\right) \tilde{B}_{n-k-1}^{(k+1)}\right) B_{k-1}
$$

$$
\text { for } n=0,1,2, \ldots
$$


This can be proved by manipulating the recurrence relations for $B_{n}$ and $\tilde{B}_{n}$. (See [10].)

One can also derive similar results for the numerators $A_{n}$, but that is not necessary here, since we always have

$$
A_{n}=a_{1} B_{n-1}^{(1)} \text {. }
$$

Lemma 2.3. Let $M>0, p_{n} \geq 0$ and $q_{n} \geq 0$ satisfy

$$
p_{n} \leq M+\sum_{k=0}^{n-1} q_{k} p_{k} \text { for } n=0,1,2, \ldots
$$

Then

$$
p_{n} \leq M \prod_{k=0}^{n-1}\left(1+q_{k}\right) \leq M \exp \left(\sum_{k=0}^{n-1} q_{k}\right) \text { for } n=0,1,2, \ldots
$$

This result, which essentially can be found in [1, p. 455], can be proved by induction, using that $\exp (q) \geq 1+q$ for $q \geq 0$.

Let us now turn to the more special case where $K\left(a_{n} / b_{n}\right)$ is limit periodic of elliptic type. We shall use the fact that $K\left(a_{n} / b_{n}\right)$ behaves almost like the corresponding periodic continued fraction $K(a / b)$ under our conditions. For the periodic one we have:

Lemma 2.4. Let $\left\{\tilde{B}_{n}\right\}$ be the denominators of the periodic continued fraction $K(a / b)$ of elliptic type, and let $x$ be a fixed point of the corresponding elliptic linear fractional transformation (1.1). Then $\left\{\tilde{B}_{n} \cdot(-x)^{-n}\right\}_{n=-1}^{\infty}$ is bounded and diverges by oscillation. In fact,

$$
\frac{\tilde{B}_{n}}{(-x)^{n}}=\sum_{j=0}^{n}\left(\frac{b+x}{-x}\right)^{j}=\frac{1-\left(\frac{b+x}{-x}\right)^{n+1}}{1-\frac{b+x}{-x}},
$$

where $|(b+x) / x|=1$, but $(b+x) / x \neq-1$.

Proof. The formula (2.7) is a simple consequence of (2.1), if one uses the fact that the constant sequence $\{x\}$ clearly is a tail sequence for $K(a / b)$. The rest then follows by (1.2).

This leads to the following result for our limit periodic continued fraction:

Lemma 2.5. Let $K\left(a_{n} / b_{n}\right)$ be as in Theorem 1.1 with denominators $\left\{B_{n}\right\}$, let $\left\{B_{n}^{(m)}\right\}$ be the denominators of its mth tail, and let $x$ be a fixed point of the corresponding elliptic transformation (1.1). Then:

A. The sequences $\left\{B_{n}^{(m)} \cdot(-x)^{-n}\right\}_{n=0}^{\infty}$ are bounded, uniformly with respect to $m$.

B. At least one of the two sequences $\left\{B_{n} \cdot(-x)^{-n}\right\}$ and $\left\{B_{n}^{(1)} \cdot(-x)^{-n}\right\}$ diverges by oscillation.

Proof. A. We use Lemma 2.2 with $K(a / b)$ as $K\left(\tilde{a}_{n} / \tilde{b}_{n}\right)$. This gives

$$
\frac{B_{n}}{(-x)^{n}}=\frac{\tilde{B}_{n}}{(-x)^{n}}+\sum_{k=1}^{n}\left(\frac{b_{k}-b}{-x} \frac{\tilde{B}_{n-k}}{(-x)^{n-k}}+\frac{a_{k+1}-a}{x^{2}} \frac{\tilde{B}_{n-k-1}}{(-x)^{n-k-1}}\right) \frac{B_{k-1}}{(-x)^{k-1}},
$$


where we have used that $\tilde{B}_{m}^{(k)}=\tilde{B}_{m}$ since $K(a / b)$ is periodic. By Lemma 2.4 we know that

$$
\left|\frac{\tilde{B}_{m}}{(-x)^{m}}\right| \leq \frac{2}{\left|1-\frac{b+x}{-x}\right|}=: M<\infty \quad \text { for all } m .
$$

Hence,

$$
\left|\frac{B_{n}}{(-x)^{n}}\right| \leq M+\sum_{k=1}^{n}\left(\left|\frac{b_{k}-b}{-x}\right|+\left|\frac{a_{k+1}-a}{x^{2}}\right|\right) M\left|\frac{B_{k-1}}{(-x)^{k-1}}\right|,
$$

so by Lemma 2.3 ,

$$
\begin{aligned}
\left|\frac{B_{n}}{(-x)^{n}}\right| & \leq M \exp \left(\sum_{k=1}^{n}\left(\left|\frac{b_{k}-b}{-x}\right|+\left|\frac{a_{k+1}-a}{x^{2}}\right|\right) M\right) \\
& \leq M \exp \left(M \sum_{k=1}^{\infty}\left(\left|\frac{b_{k}-b}{-x}\right|+\left|\frac{a_{k+1}-a}{x^{2}}\right|\right)\right)=: M_{1} .
\end{aligned}
$$

Similarly,

$$
\left|\frac{B_{n}^{(m)}}{(-x)^{n}}\right| \leq M \exp \left(M \sum_{k=m+1}^{\infty}\left(\left|\frac{b_{k}-b}{-x}\right|+\left|\frac{a_{k+1}-a}{x^{2}}\right|\right)\right) \leq M_{1}
$$

for all $n \geq 1$ and $m \geq 0$.

B. To prove the oscillation, we insert (2.7) into (2.8). For convenience we write

$$
\delta=(b+x) /(-x)
$$

Then (2.8) can be written

$$
\begin{aligned}
\frac{B_{n}}{(-x)^{n}}= & \frac{1-\delta^{n+1}}{1-\delta}+\sum_{k=1}^{n}\left(\frac{b_{k}-b}{-x} \cdot \frac{1-\delta^{n-k+1}}{1-\delta}+\frac{a_{k+1}-a}{x^{2}} \cdot \frac{1-\delta^{n-k}}{1-\delta}\right) \frac{B_{k-1}}{(-x)^{k-1}} \\
= & \frac{1}{1-\delta}\left\{1+\sum_{k=1}^{n}\left(\frac{b_{k}-b}{-x}+\frac{a_{k+1}-a}{x^{2}}\right) \frac{B_{k-1}}{(-x)^{k-1}}\right\} \\
& -\frac{\delta^{n+1}}{1-\delta}\left\{1+\sum_{k=1}^{n}\left(\frac{b_{k}-b}{-x} \delta^{-k}+\frac{a_{k+1}-a}{x^{2}} \delta^{-k-1}\right) \frac{B_{k-1}}{(-x)^{k-1}}\right\},
\end{aligned}
$$

where both series in the last expression converge absolutely as $n \rightarrow \infty$. Hence, the $\delta^{n+1}$-term gives the oscillation unless

$$
1+\sum_{k=1}^{\infty}\left(\frac{b_{k}-b}{-x} \delta^{-k}+\frac{a_{k+1}-a}{x^{2}} \delta^{-k-1}\right) \frac{B_{k-1}}{(-x)^{k-1}}=0 .
$$

Assume that $(2.15)$ holds. Then $\left\{B_{n} \cdot(-x)^{-n}\right\}$ converges to a finite number. Assume that also $\left\{B_{n}^{(1)} \cdot(-x)^{-n}\right\}$ converges to a finite number. Then $\left\{B_{n}^{(m)} \cdot(-x)^{-n}\right\}$ converge to finite numbers for all $m \in \mathbf{N}$, since by induction

$$
B_{n}^{(m)}=b_{m+1} B_{n-1}^{(m+1)}+a_{m+2} B_{n-2}^{(m+2)} .
$$


That is, by the arguments above,

$$
1+\sum_{k=1}^{\infty}\left(\frac{b_{k+m}-b}{-x} \delta^{-k}+\frac{a_{k+m+1}-a}{x^{2}} \delta^{-k-1}\right) \frac{B_{k-1}^{(m)}}{(-x)^{k-1}}=0
$$

for all $m \in \mathbf{N}$. This is impossible since (1.4) holds, $|\delta|=1$, and $\left\{B_{k-1}^{(m)} \cdot(-x)^{-k+1}\right\}$ is bounded, uniformly with respect to $m$. Hence, if $\left\{B_{n} \cdot(-x)^{-n}\right\}$ converges, then $\left\{B_{n}^{(1)} \cdot(-x)^{-n}\right\}$ oscillates.

Note that the oscillation has the character as described in Remark 3.

Proof of Theorem 1.1. We shall first prove part B, then part A and finally part C.

B. From Lemma 2.2 with $K\left(\tilde{a}_{n} / \tilde{b}_{n}\right)$ equal to the periodic continued fraction $K(a / b)$, we find that

$$
\begin{aligned}
B_{n}+B_{n-1} x= & \left(\tilde{B}_{n}+\tilde{B}_{n-1} x\right) \\
& +\sum_{k=1}^{n-1}\left(\left(b_{k}-b\right)\left(\tilde{B}_{n-k}+\tilde{B}_{n-k-1} x\right)\right. \\
& \left.\quad+\left(a_{k+1}-a\right)\left(\tilde{B}_{n-k-1}+\tilde{B}_{n-k-2} x\right)\right) B_{k-1} \\
& +\left(b_{n}-b\right) B_{n-1},
\end{aligned}
$$

where we again have used that $\tilde{B}_{m}^{(k)}=\tilde{B}_{m}$. It follows easily by induction (or from Lemma 2.1) that

$$
\tilde{B}_{n}+\tilde{B}_{n-1} x=(b+x)^{n} \quad \text { for } n=0,1,2, \ldots
$$

Hence,

$$
\begin{aligned}
\frac{B_{n}+B_{n-1} x}{(b+x)^{n}}= & 1+\sum_{k=1}^{n-1}\left(\frac{b_{k}-b}{b+x}+\frac{a_{k+1}-a}{(b+x)^{2}}\right) \frac{B_{k-1}}{(b+x)^{k-1}} \\
& +\frac{b_{n}-b}{b+x} \frac{B_{n-1}}{(b+x)^{n-1}} .
\end{aligned}
$$

From Lemma $2.5 \mathrm{~A}$ we know that $\left\{B_{k}(b+x)^{-k}\right\}$ is a bounded sequence since also $-(b+x)$ is a fixed point of (1.1). Hence, the series in (2.20) converges absolutely as $n \rightarrow \infty$ and the last term vanishes. Using (2.4), we similarly find that

$$
\begin{array}{r}
\frac{A_{n}+A_{n-1} x}{(b+x)^{n}}=\frac{a_{1}}{b+x}\left(1+\sum_{k=1}^{n-2}\left(\frac{b_{k+1}-b}{b+x}+\frac{a_{k+2}-a}{(b+x)^{2}}\right) \frac{B_{k-1}^{(1)}}{(b+x)^{k-1}}\right. \\
\left.+\frac{b_{n}-b}{b+x} \frac{B_{n-2}^{(1)}}{(b+x)^{n-2}}\right)
\end{array}
$$

converges to a finite value as $n \rightarrow \infty$.

We want to prove that the limits of $(2.20)$ and (2.21) are not both equal to zero. So assume that

$$
\lim _{n \rightarrow \infty} \frac{B_{n}+B_{n-1} x}{(b+x)^{n}}=\lim _{n \rightarrow \infty} \frac{A_{n}+A_{n-1} x}{(b+x)^{n}}=\frac{a_{1}}{b+x} \lim _{n \rightarrow \infty} \frac{B_{n}^{(1)}+B_{n-1}^{(1)} x}{(b+x)^{n}}=0
$$


That is, by (2.20) and (2.21),

$$
1+\sum_{k=1}^{\infty}\left(\frac{b_{k+m}-b}{b+x}+\frac{a_{k+m+1}-a}{(b+x)^{2}}\right) \frac{B_{k-1}^{(m)}}{(b+x)^{k-1}}=0
$$

for $m=0$ and $m=1$. From (2.16) we find that

$$
B_{n}^{(m)}+B_{n-1}^{(m)} x=b_{m+1}\left(B_{n-1}^{(m+1)}+B_{n-2}^{(m+1)} x\right)+a_{m+2}\left(B_{n-2}^{(m+2)}+B_{n-3}^{(m+2)} x\right),
$$

which implies that (2.23) holds for all $m \geq 0$. This is impossible because of (2.12). Hence, the convergence of $S_{n}(x)$ to a value in $\hat{\mathbf{C}}$ follows from (0.4).

A. We want to prove that $S_{n}(0)=A_{n} / B_{n}$ diverges in $\hat{\mathbf{C}}$ (i.e., oscillates) as $n \rightarrow \infty$. Since $S_{n}(x)$ converges, it suffices to prove that $\left(S_{n}(x)-S_{n-1}(0)\right)$ oscillates.

Without loss of generality we assume that $\left\{B_{n} \cdot(-x)^{-n}\right\}$ diverges by oscillation and that the limit of (2.20) is nonzero. (The limit $\lim _{n \rightarrow \infty}\left(B_{n}^{(m)}+B_{n-1}^{(m)} x\right)(b+x)^{-n}$ is clearly nonzero from some $m$ on (see (2.20)), say for $m \geq m_{0}$, and if $\left\{B_{n}^{\left(m_{0}\right)} \cdot(-x)^{-n}\right\}$ converges, then $\left\{B_{n}^{\left(m_{0}+1\right)} \cdot(-x)^{-n}\right\}$ oscillates. (See Lemma 2.5B.) Finally, $K\left(a_{n} / b_{n}\right)$ converges if and only if its $k$ th tail converges, $k=m_{0}$ or $\left.k=m_{0}+1.\right)$

We have, using the determinant formula $(0.5)$,

$$
\begin{aligned}
S_{n}(x)-S_{n-1}(0) & =\frac{A_{n}+A_{n-1} x}{B_{n}+B_{n-1} x}-\frac{A_{n-1}}{B_{n-1}} \\
& =\frac{A_{n} B_{n-1}-B_{n} A_{n-1}}{B_{n-1}\left(B_{n}+B_{n-1} x\right)}=x \frac{\prod_{k=1}^{n}\left(1+\frac{a_{k}-a}{a}\right)}{\frac{B_{n-1}}{(-x)^{n-1}} \cdot \frac{B_{n}+B_{n-1} x}{(b+x)^{n}}},
\end{aligned}
$$

since $a=x(b+x)$. The numerator of this last expression converges to a finite value $\neq 0$ as $n \rightarrow \infty$ because of (1.4), and so does also the second factor of its denominator. Since $\left\{B_{n-1} \cdot(-x)^{-n+1}\right\}$ diverges by oscillation, the oscillation of the whole expression follows. Note that the oscillation is as described in Remark 3.

C. Let $\left\{t_{n}\right\}$ be the tail sequence of $K\left(a_{n} / b_{n}\right)$ with $t_{0}=\lim _{n \rightarrow \infty} S_{n}(x)$. Then

$$
\begin{aligned}
t_{m} & =\lim _{n \rightarrow \infty} S_{n}^{(m)}(x)=\lim _{n \rightarrow \infty} \frac{A_{n}^{(m)}+A_{n-1}^{(m)} x}{B_{n}^{(m)}+B_{n-1}^{(m)} x} \\
& =\frac{a_{m+1}}{b+x} \cdot \frac{1+\sum_{k=1}^{\infty} d_{m+k+1} D_{k-1}^{(m+1)}}{1+\sum_{k=1}^{\infty} d_{m+k} D_{k-1}^{(m)}},
\end{aligned}
$$

where the last equality follows from (2.20) and (2.21) when

$$
d_{j}=\frac{b_{j}-b}{b+x}+\frac{a_{j+1}-a}{(b+x)^{2}}, \quad D_{j}^{(m)}=\frac{B_{j}^{(m)}}{(b+x)^{j}} .
$$

Now, $\sum\left|d_{j}\right|<\infty$, and $\left\{D_{j}^{(m)}\right\}_{j=0}^{\infty}$ is uniformly bounded with respect to $m$. (See Lemma 2.5A.) Hence, as $m \rightarrow \infty$ the first factor in (2.26) approaches $a /(b+x)=x$ and the second factor approaches 1 . 
Proof of Theorem 1.2. A. We first observe that $a_{n} \rightarrow-1 / 4$, so that $K\left(a_{n} z / 1\right)$ is limit periodic of elliptic type for $z>1$. Hence, it would suffice to prove that $\sum\left|a_{n}+1 / 4\right|<\infty$. However, it is readily seen that this does not hold in general for the Gauss fraction.

On the other hand, it suffices to prove that the even or the odd part of $1+K\left(a_{n} z / 1\right)$ diverges, i.e., that $\left\{S_{2 n}(0)\right\}$ or $\left\{S_{2 n+1}(0)\right\}$ diverges. A continued fraction which has classical approximants equal to $\left\{S_{2 n}(0)\right\}$ is given by

$$
1+\frac{a_{1} z}{1+a_{2} z}-\frac{a_{2} a_{3} z^{2}}{1+a_{3} z+a_{4} z}-\frac{a_{4} a_{5} z^{2}}{1+a_{5} z+a_{6} z}-\frac{a_{6} a_{7} z^{2}}{1+a_{7} z+a_{8} z}-\ldots
$$

(See, for instance, $[8, \mathrm{p} .42]$.)

Let us first assume that $z \neq 2$. Then $1+a_{2 n-1} z+a_{2 n} z \neq 0$ from some $n$ on. Since (2.28) diverges if and only if a tail of (2.28) diverges, we may without loss of generality assume that $1+a_{2} z \neq 0$ and $1+a_{2 n-1} z+a_{2 n} z \neq 0$ for all $n \geq 2$. An equivalence transformation (see, for instance, $[8, \mathrm{p} .31]$ ) will then bring (2.28) over to the form $1+K\left(c_{n}(z) / 1\right)$, where

$$
c_{n+1}(z)=\frac{-a_{2 n} a_{2 n+1} z^{2}}{\left(1+a_{2 n-1} z+a_{2 n} z\right)\left(1+a_{2 n+1} z+a_{2 n+2} z\right)}
$$

for $n \geq 2$. Inserting the expressions (1.7) for $\left\{a_{n}\right\}$ into (2.29), we find (after some computation) that

$$
\begin{aligned}
c_{n+1}(z)= & -\frac{z^{2}}{4(2-z)^{2}}+ \\
& +\frac{1}{16 n^{2}} \frac{z^{2}}{(2-z)^{3}}\left(2(c-2 a)^{2}+2(c-2 b)^{2}-2-z\left(4(a-b)^{2}-1\right)\right) \\
& +\mathscr{O}\left(n^{-3}\right) .
\end{aligned}
$$

For $z>1, z \neq 2$ we find that $-z^{2} / 4(2-z)^{2}<-1 / 4$, so that also $K\left(c_{n}(z) / 1\right)$ is limit periodic of elliptic type, and the divergence of $K\left(c_{n}(z) / 1\right)$ follows from Theorem 1.1A.

For $z=2$ we have

$$
\begin{aligned}
1+ & a_{2 n-1} z+a_{2 n} z \\
& =1-2\left(\frac{(a+n-1)(c-b+n-1)}{(c+2 n-2)(c+2 n-1)}+\frac{(b+n)(c-a+n)}{(c+2 n-1)(c+2 n)}\right) \\
& =\frac{(c-2 a)(c-2 b)(2 n+c-1)}{(c+2 n-2)(c+2 n-1)(c+2 n)}=\frac{(c-2 a)(c-2 b)}{(c+2 n-2)(c+2 n)} .
\end{aligned}
$$

Hence, if $c=2 a$ or $c=2 b$, then the first tail of $(2.28)$ has the form $K\left(d_{n} / 0\right)$. This is a divergent continued fraction since its sequence of classical approximants $\left\{A_{n} / B_{n}\right\}$ has the form $\infty, 0, \infty, 0, \infty, 0, \ldots$.

Let $c \neq 2 a$ and $c \neq 2 b$. Then (2.28) has the form $1+K\left(d_{n} / e_{n}\right)$, where $d_{n} \rightarrow-1 / 4$ and $e_{n}$ is given by $(2.31)$, i.e., $e_{n}=\mathscr{O}\left(n^{-2}\right)$, which means that $K\left(d_{n} / e_{n}\right)$ diverges by virtue of the classical Stern-Stolz Theorem [8, p. 79].

B. For $z=1$ the expression (2.30) reduces to

$$
c_{n+1}(1)=-\frac{1}{4}+\frac{4(c-a-b)^{2}-1}{16 n^{2}}+\mathscr{O}\left(n^{-3}\right)
$$


and thus, for $c-a-b=i t$, it has the form $c_{n+1}(1)=-1 / 4-d / 16 n^{2}+\mathscr{O}\left(n^{-3}\right)$ for a $d>1$. Hence, $K\left(c_{n}(1) / 1\right)$ diverges by Theorem 3.1 in [6].

To prove Theorem 1.3, we shall use the following lemma:

Lemma 2.6. Let $\left\{t_{n}\right\}$ be a tail sequence for $K\left(a_{n} / b_{n}\right)$ with all $t_{n} \neq \infty$. Then $K\left(a_{n} / b_{n}\right)$ converges in $\hat{\mathbf{C}}$ if and only if

$$
\sum_{n=0}^{\infty} \prod_{j=1}^{n} \frac{b_{j}+t_{j}}{-t_{j}}
$$

converges in $\hat{\mathbf{C}}$. Moreover, $K\left(a_{n} / b_{n}\right)$ converges to $t_{0}$ if and only if the sum of (2.33) is infinite.

This is a generalization of a result by Waadeland [17]. It follows readily by dividing

$$
A_{n}-B_{n} t_{0}=\prod_{m=0}^{n}\left(-t_{m}\right) \quad \text { (proved by induction) }
$$

by $B_{n}$ as given by (2.1), and letting $n \rightarrow \infty$.

Proof of Theorem 1.3. The sequence $\left\{t_{n}\right\}$ given by $(1.10)$ is a tail sequence for $K\left(a_{n} / 1\right)$ with all $t_{n} \neq \infty$. The sum (2.33) can be written

$$
\begin{aligned}
\sum_{n=0}^{\infty} & \prod_{j=1}^{n} \frac{\frac{1}{2}+i t+r /(j+1)}{\frac{1}{2}-i t-r /(j+1)} \\
& =\sum_{n=0}^{\infty} \prod_{j=1}^{n}\left(\frac{\frac{1}{2}+i t}{\frac{1}{2}-i t}+\frac{r /(j+1)}{\left(\frac{1}{2}-i t\right)^{2}}+\mathscr{O}\left(j^{-2}\right)\right) \\
& =\sum_{n=0}^{\infty}\left(\frac{\frac{1}{2}+i t}{\frac{1}{2}-i t}\right)^{n} \prod_{j=1}^{n}\left(1+\frac{r /(j+1)}{\frac{1}{4}+t^{2}}+\mathscr{O}\left(j^{-2}\right)\right) \\
& =\sum_{n=0}^{\infty} e^{2 i n \theta} \prod_{j=1}^{n}\left(1-\frac{r}{a} \cdot \frac{1}{j}+\mathscr{O}\left(j^{-2}\right)\right),
\end{aligned}
$$

where $\theta=\arg \left(\frac{1}{2}+i t\right)$ and $a=-\left(t^{2}+1 / 4\right)$. According to [9, p.232]

$$
\prod_{j=1}^{n}\left(1-\frac{r}{a} \cdot \frac{1}{j}\right)=\gamma n^{-r / a}+o\left(n^{-r / a}\right) \quad \text { as } n \rightarrow \infty
$$

for a constant $\gamma \neq 0$. If $\operatorname{Re}(r)<-\left(t^{2}+1 / 4\right)$, then the sum (2.35) converges absolutely (to a finite value).

\section{A GENERALIZATION}

The ideas leading to Theorem 1.1 can also be used in the more general situation where $K\left(a_{n} / b_{n}\right)$ is "close to" some continued fraction $K\left(\tilde{a}_{n} / \tilde{b}_{n}\right)$, even 
if $K\left(\tilde{a}_{n} / \tilde{b}_{n}\right)$ is not periodic as $K(a / b)$ is. In the periodic case we always had (Lemma 2.4) that

$$
\left|\frac{\tilde{B}_{n}^{(j)}}{(-x)^{n}}\right| \leq M \quad \text { and } \quad\left|\frac{\tilde{B}_{n}^{(j)}}{(b+x)^{n}}\right| \leq M \quad \text { for all } n \geq 0, \quad j \geq 0
$$

for some finite constant $M>0$. The analogue to this is now

$$
\left|\frac{\tilde{B}_{n}^{(j)}}{\prod_{m=j+1}^{j+n}\left(-\tilde{t}_{m}\right)}\right| \leq M \quad \text { and } \quad\left|\frac{\tilde{B}_{n}^{(j)}}{\prod_{m=j+1}^{j+n}\left(\tilde{b}_{m}+\tilde{t}_{m}\right)}\right| \leq M \quad \text { for } n \geq 0, j \geq 0,
$$

where $\left\{\tilde{t}_{n}\right\}, \tilde{t}_{n} \neq \infty$, is a tail sequence for $K\left(\tilde{a}_{n} / \tilde{b}_{n}\right)$ and $\left\{\tilde{B}_{n}^{(j)}\right\}_{n=-1}^{\infty}$ are the denominators of its $j$ th tail. Hence, we shall require that (3.2) holds. Note that by Lemma 2.1 this condition on $K\left(\tilde{a}_{n} / \tilde{b}_{n}\right)$ can be written

$$
\left|\sum_{k=0}^{n} \prod_{m=j+1}^{j+k} \frac{\tilde{b}_{m}+\tilde{t}_{m}}{-\tilde{t}_{m}}\right| \leq M \text { and }\left|\sum_{k=0}^{n} \prod_{m=j+k+1}^{j+n} \frac{-\tilde{t}_{m}}{\tilde{b}_{m}+\tilde{t}_{m}}\right| \leq M .
$$

Since

$$
\prod_{m=j+1}^{j+n} \frac{\tilde{b}_{m}+\tilde{t}_{m}}{-\tilde{t}_{m}}=\sum_{k=0}^{n} \prod_{m=j+1}^{j+k} \frac{\tilde{b}_{m}+\tilde{t}_{m}}{-\tilde{t}_{m}}-\sum_{k=0}^{n-1} \prod_{m=j+1}^{j+k} \frac{\tilde{b}_{m}+\tilde{t}_{m}}{-\tilde{t}_{m}}
$$

and

$$
\prod_{m=j+1}^{j+n} \frac{-\tilde{t}_{m}}{\tilde{b}_{m}+\tilde{t}_{m}}=\sum_{k=0}^{n} \prod_{m=j+k+1}^{j+n} \frac{-\tilde{t}_{m}}{\tilde{b}_{m}+\tilde{t}_{m}}-\sum_{k=0}^{n-1} \prod_{m=(j+1)+k+1}^{(j+1)+n-1} \frac{-\tilde{t}_{m}}{\tilde{b}_{m}+\tilde{t}_{m}}
$$

this actually means that

$$
\frac{1}{2 M} \leq\left|\prod_{m=j+1}^{j+n} \frac{\tilde{b}_{m}+\tilde{t}_{m}}{-\tilde{t}_{m}}\right| \leq 2 M \quad \text { for all } j \geq 0, n \geq 0 .
$$

Hence, the first sum in $\left(3.2^{\prime}\right)$ cannot converge in $\hat{\mathbf{C}}$, so by Lemma $2.6, K\left(\tilde{a}_{n} / \tilde{b}_{n}\right)$ has to diverge.

Next we shall impose conditions on the "closeness" of $K\left(a_{n} / b_{n}\right)$ to $K\left(\tilde{a}_{n} / \tilde{b}_{n}\right)$ :

$$
\sum_{n=1}^{\infty}\left|\frac{b_{n}-\tilde{b}_{n}}{\tilde{b}_{n}}\right|<\infty \text { and } \sum_{n=1}^{\infty}\left|\frac{a_{n+1}-\tilde{a}_{n+1}}{\tilde{b}_{n} \tilde{b}_{n+1}}\right|<\infty
$$

Observe that by $\left(3.2^{\prime}\right)$ with $n=1$ we find that

$$
\left|\frac{\tilde{b}_{j+1}}{\tilde{t}_{j+1}}\right| \leq M \quad \text { and } \quad\left|\frac{\tilde{b}_{j+1}}{\tilde{b}_{j+1}+\tilde{t}_{j+1}}\right| \leq M \quad \text { for all } j \geq 0
$$


Our conditions therefore imply that

$$
\begin{gathered}
\sum_{n=1}^{\infty}\left|\frac{b_{n}-\tilde{b}_{n}}{\tilde{b}_{n}+\tilde{t}_{n}}\right|<\infty, \quad \sum_{n=1}^{\infty}\left|\frac{a_{n+1}-\tilde{a}_{n+1}}{\left(\tilde{b}_{n}+\tilde{t}_{n}\right)\left(\tilde{b}_{n+1}+\tilde{t}_{n+1}\right)}\right|<\infty, \\
\sum_{n=1}^{\infty}\left|\frac{b_{n}-\tilde{b}_{n}}{\tilde{t}_{n}}\right|<\infty, \quad \sum_{n=1}^{\infty}\left|\frac{a_{n+1}-\tilde{a}_{n+1}}{\tilde{t}_{n} \tilde{t}_{n+1}}\right|<\infty, \\
\sum_{n=1}^{\infty}\left|\frac{a_{n+1}-\tilde{a}_{n+1}}{\tilde{t}_{n}\left(\tilde{b}_{n+1}+\tilde{t}_{n+1}\right)}\right|<\infty .
\end{gathered}
$$

Of course, for the case where $\left\{\tilde{b}_{n}\right\}$ is bounded and bounded away from the origin, such as for $K(a / b)$ in Theorem 1.1, the condition (3.6) reduces to (1.4).

Theorem 3.1. Let $K\left(\tilde{a}_{n} / \tilde{b}_{n}\right)$ be a continued fraction with tail sequence $\left\{\tilde{t}_{n}\right\}$ such that all $\tilde{t}_{n} \neq \infty$ and $\left(3.2^{\prime}\right)$ holds for all $n \geq 0$ and $j \geq 0$. Let further $K\left(a_{n} / b_{n}\right)$ be a continued fraction close to $K\left(\tilde{a}_{n} / \tilde{b}_{n}\right)$ in the sense that (3.6) holds. Then:

A. $K\left(a_{n} / b_{n}\right)$ diverges.

B. The modified approximants $S_{n}\left(\tilde{t}_{n}\right)$ of $K\left(a_{n} / b_{n}\right)$ converge to a limit $t_{0} \in \hat{\mathbf{C}}$ as $n \rightarrow \infty$.

C. The tail sequence $\left\{t_{n}\right\}$ of $K\left(a_{n} / b_{n}\right)$ with $t_{0}=\lim S_{n}\left(\tilde{t}_{n}\right)$ satisfies $\lim _{n \rightarrow \infty}\left(t_{n}-\tilde{t}_{n}\right)=0$.

Proof. By copying the proof of Lemma 2.5A we find that

$$
\begin{array}{r}
\left|\frac{B_{n}^{(j)}}{\prod_{m=j+1}^{j+n}\left(-\tilde{t}_{m}\right)}\right| \leq M_{1} \text { and }\left|\frac{B_{n}^{(j)}}{\prod_{m=j+1}^{j+n}\left(\tilde{b}_{m}+\tilde{t}_{m}\right)}\right| \leq M_{1} \\
\text { for } n \geq 1, \quad j \geq 0
\end{array}
$$

for some finite constant $M_{1}$. We also want to prove that the two sequences $\left\{B_{n} / \prod_{m=1}^{n}\left(-\tilde{t}_{m}\right)\right\}$ and $\left\{B_{n}^{(1)} / \prod_{m=2}^{n+1}\left(-\tilde{t}_{m}\right)\right\}$ cannot both converge in $\hat{\mathbf{C}}$ as $n \rightarrow$ $\infty$. To do this, we copy the proof of Lemma $2.5 \mathrm{~B}$. But first we observe that by Lemma 2.1

$$
\begin{aligned}
B_{n}= & \sum_{k=0}^{n}\left(\prod_{m=1}^{k}\left(b_{m}+t_{m}\right) \prod_{m=k+1}^{n}\left(-t_{m}\right)\right) \\
= & \sum_{k=0}^{j-1}\left(\prod_{m=1}^{k}\left(b_{m}+t_{m}\right) \prod_{m=k+1}^{n}\left(-t_{m}\right)\right) \\
& +\sum_{k=j}^{n}\left(\prod_{m=1}^{k}\left(b_{m}+t_{m}\right) \prod_{m=k+1}^{n}\left(-t_{m}\right)\right) \\
= & B_{j-1} \prod_{m=j}^{n}\left(-t_{m}\right)+B_{n-j}^{(j)} \prod_{m=1}^{j}\left(b_{m}+t_{m}\right)
\end{aligned}
$$


for $0 \leq j \leq n$. Combining this with Lemma 2.2 gives

(3.11)

$$
\begin{aligned}
& \frac{B_{n}}{\prod_{m=1}^{n}\left(-\tilde{t}_{m}\right)}=\frac{\tilde{B}_{n}}{\prod_{m=1}^{n}\left(-\tilde{t}_{m}\right)} \\
& +\sum_{k=1}^{n}\left(\frac{b_{k}-\tilde{b}_{k}}{-\tilde{t}_{k}} \cdot \frac{\tilde{B}_{n-k}^{(k)}}{\prod_{m=k+1}^{n}\left(-\tilde{t}_{m}\right)}+\frac{a_{k+1}-\tilde{a}_{k+1}}{\tilde{t}_{k} \tilde{t}_{k+1}} \cdot \frac{\tilde{B}_{n-k-1}^{(k+1)}}{\prod_{m=k+2}^{n}\left(-\tilde{t}_{m}\right)}\right) \frac{B_{k-1}}{\prod_{m=1}^{k-1}\left(-\tilde{t}_{m}\right)} \\
& =\frac{\tilde{B}_{n}}{\prod_{m=1}^{n}\left(-\tilde{t}_{m}\right)}+\sum_{k=1}^{n}\left(\frac{b_{k}-\tilde{b}_{k}}{-\tilde{t}_{k}} \cdot \frac{\tilde{B}_{n}-\tilde{B}_{k-1} \prod_{m=k}^{n}\left(-\tilde{t}_{m}\right)}{\prod_{m=1}^{k}\left(\tilde{b}_{m}+\tilde{t}_{m}\right) \prod_{m=k+1}^{n}\left(-\tilde{t}_{m}\right)}\right. \\
& \left.+\frac{a_{k+1}-\tilde{a}_{k+1}}{\tilde{t}_{k} \tilde{t}_{k+1}} \cdot \frac{\tilde{B}_{n}-\tilde{B}_{k} \prod_{m=k+1}^{n}\left(-\tilde{t}_{m}\right)}{\prod_{m=1}^{k+1}\left(\tilde{b}_{m}+\tilde{t}_{m}\right) \prod_{m=k+2}^{n}\left(-\tilde{t}_{m}\right)}\right) \frac{B_{k-1}}{\prod_{m=1}^{k-1}\left(-\tilde{t}_{m}\right)} \\
& =\frac{\tilde{B}_{n}}{\prod_{m=1}^{n}\left(-\tilde{t}_{m}\right)}\left\{1+\sum_{k=1}^{n}\left(\frac{b_{k}-\tilde{b}_{k}}{-\tilde{t}_{k}} \prod_{m=1}^{k} \frac{-\tilde{t}_{m}}{\tilde{b}_{m}+\tilde{t}_{m}}\right.\right. \\
& \left.\left.+\frac{a_{k+1}-\tilde{a}_{k+1}}{\tilde{t}_{k} \tilde{t}_{k+1}} \prod_{m=1}^{k+1} \frac{-\tilde{t}_{m}}{\tilde{b}_{m}+\tilde{t}_{m}}\right) \frac{B_{k-1}}{\prod_{m=1}^{k-1}\left(-\tilde{t}_{m}\right)}\right\} \\
& -\sum_{k=1}^{n}\left(\frac{b_{k}-\tilde{b}_{k}}{\tilde{b}_{k}+\tilde{t}_{k}} \cdot \frac{\tilde{B}_{k-1}}{\prod_{m=1}^{k-1}\left(\tilde{b}_{m}+\tilde{t}_{m}\right)}\right. \\
& \left.+\frac{a_{k+1}-\tilde{a}_{k+1}}{-\tilde{t}_{k}\left(\tilde{b}_{k+1}+\tilde{t}_{k+1}\right)} \cdot \frac{\tilde{B}_{k}}{\prod_{m=1}^{k}\left(\tilde{b}_{m}+\tilde{t}_{m}\right)}\right) \frac{B_{k-1}}{\prod_{m=1}^{k-1}\left(-\tilde{t}_{m}\right)} .
\end{aligned}
$$

The second series in this last expression converges absolutely as $n \rightarrow \infty$ since (3.2), (3.8), and (3.9) hold. So does also the first series since by (3.5)

$$
\left|\prod_{m=1}^{k} \frac{-\tilde{t}_{m}}{\tilde{b}_{m}+\tilde{t}_{m}}\right| \leq 2 M .
$$

Since $K\left(\tilde{a}_{n} / \tilde{b}_{n}\right)$ diverges, we know by Lemma 2.6 that $\left\{\tilde{B}_{n} / \prod_{m=1}^{n}\left(-\tilde{t}_{m}\right)\right\}$ diverges, and the question is again reduced to the question of whether

$$
\begin{aligned}
1+\sum_{k=1}^{\infty}( & \frac{b_{k+j}-\tilde{b}_{k+j}}{-\tilde{t}_{k+j}} \prod_{m=1}^{k} \frac{-\tilde{t}_{m+j}}{\tilde{b}_{m+j}+\tilde{t}_{m+j}} \\
& \left.\quad+\frac{a_{k+j+1}-\tilde{a}_{k+j+1}}{\tilde{t}_{k+j} \tilde{t}_{k+j+1}} \prod_{m=1}^{k+1} \frac{-\tilde{t}_{m+j}}{\tilde{b}_{m+j}+\tilde{t}_{m+j}}\right) \frac{B_{k-1}^{(j)}}{\prod_{m=1}^{k-1}\left(-\tilde{t}_{m+j}\right)}=0
\end{aligned}
$$

can be true simultaneously for $j=0$ and $j=1$. But, just as in Lemma $2.5 \mathrm{~B}$, if (3.13) holds for $j=0,1$, then (3.13) holds for all $j \geq 0$, which is impossible. Hence, the oscillation follows. Note also that this oscillation has the same character as the one of $\tilde{B}_{n} / \prod_{m=1}^{n}\left(-\tilde{t}_{m}\right)$.

Let us start by proving part $B$ :

B. The analogue to $(2.18)$ is 


$$
\begin{aligned}
B_{n}+B_{n-1} \tilde{t}_{n}= & \left(\tilde{B}_{n}+\tilde{B}_{n-1} \tilde{t}_{n}\right) \\
& +\sum_{k=1}^{n-1}\left(\left(b_{k}-\tilde{b}_{k}\right)\left(\tilde{B}_{n-k}^{(k)}+\tilde{B}_{n-k-1}^{(k)} \tilde{t}_{n}\right)\right. \\
& \left.\quad+\left(a_{k+1}-\tilde{a}_{k+1}\right)\left(\tilde{B}_{n-k-1}^{(k+1)}+\tilde{B}_{n-k-2}^{(k+1)} \tilde{t}_{n}\right)\right) B_{k-1} \\
& +\left(b_{n}-\tilde{b}_{n}\right) B_{n-1},
\end{aligned}
$$

where, by induction on $n$,

$$
\tilde{B}_{n}^{(k)}+\tilde{B}_{n-1}^{(k)} \tilde{t}_{k+n}=\prod_{m=k+1}^{k+n}\left(\tilde{b}_{m}+\tilde{t}_{m}\right) .
$$

Hence,

$$
\begin{aligned}
\frac{B_{n}+B_{n-1} \tilde{t}_{n}}{\prod_{m=1}^{n}\left(\tilde{b}_{m}+\tilde{t}_{m}\right)} \\
=1+\sum_{k=1}^{n-1}\left(\frac{b_{k}-\tilde{b}_{k}}{\tilde{b}_{k}+\tilde{t}_{k}}+\frac{a_{k+1}-\tilde{a}_{k+1}}{\left(\tilde{b}_{k}+\tilde{t}_{k}\right)\left(\tilde{b}_{k+1}+\tilde{t}_{k+1}\right)}\right) \frac{B_{k-1}}{\prod_{m=1}^{k-1}\left(\tilde{b}_{m}+\tilde{t}_{m}\right)} \\
\quad+\frac{b_{n}-\tilde{b}_{n}}{\tilde{b}_{n}+\tilde{t}_{n}} \frac{B_{n-1}}{\prod_{m=1}^{n-1}\left(\tilde{b}_{m}+\tilde{t}_{m}\right)},
\end{aligned}
$$

and the convergence of (3.16) to a finite value as $n \rightarrow \infty$ follows. The convergence of

$$
\frac{A_{n}+A_{n-1} \tilde{t}_{n}}{\prod_{m=1}^{n}\left(\tilde{b}_{m}+\tilde{t}_{m}\right)}=\frac{a_{1}}{\tilde{b}_{1}+\tilde{t}_{1}} \frac{B_{n-1}^{(1)}+B_{n-2}^{(1)} \tilde{t}_{n}}{\prod_{m=2}^{n}\left(\tilde{b}_{m}+\tilde{t}_{m}\right)}
$$

follows similarly. That the limits of (3.16) and (3.17) are not both zero follows as in the proof of Theorem 1.1B.

A. The analogue to $(2.25)$ is

$$
\begin{aligned}
S_{n}\left(\tilde{t}_{n}\right) & -S_{n-1}(0)=\frac{(-1)^{n+1} \prod_{k=1}^{n} a_{k}}{B_{n-1}\left(B_{n}+B_{n-1} \tilde{t}_{n}\right)} \\
& =\tilde{t}_{0} \frac{\prod_{k=1}^{n}\left(1+\left(a_{k}-\tilde{a}_{k}\right) / \tilde{a}_{k}\right)}{\left(B_{n-1} / \prod_{m=1}^{n-1}\left(-\tilde{t}_{m}\right)\right) \cdot\left(B_{n}+B_{n-1} \tilde{t}_{n}\right) / \prod_{m=1}^{n}\left(\tilde{b}_{m}+\tilde{t}_{m}\right)},
\end{aligned}
$$

since $\tilde{a}_{k}=\tilde{t}_{k-1}\left(\tilde{b}_{k}+\tilde{t}_{k}\right)$. We have just proved that the second factor in the denominator of this expression converges to a finite value. Without loss of generality we may assume that it is nonzero. The numerator converges to a value in $\mathbf{C} \backslash\{0\}$ by virtue of (3.8). Finally, without loss of generality, the first factor of the denominator oscillates in $\hat{\mathbf{C}}$. Observe that the oscillation of $S_{n}(0)$ thus has the same character as the one of $\tilde{S}_{n}(0)$.

C. The proof is just as for Theorem 1.1C, since

$$
\frac{a_{m}-\tilde{a}_{m}}{\tilde{b}_{m}+\tilde{t}_{m}}=\frac{a_{m}}{\tilde{b}_{m}+\tilde{t}_{m}}-\tilde{t}_{m-1} \rightarrow 0 \text {. }
$$

Also here we have actually proved that (3.16) and (3.17) converge separately to finite values which are not both equal to zero. 
Example. Let $x \neq 0,-1$ and $y \neq 0,-1$ be two complex numbers such that

$$
\frac{x(1+y)}{y(1+x)}=e^{i \theta} \quad \text { for some } 0<\theta<2 \pi .
$$

Then the 2-periodic continued fraction

$$
K \frac{\tilde{a}_{n}}{1}=-\frac{x y}{1}-\frac{(1+x)(1+y)}{1}-\frac{x y}{1}-\frac{(1+x)(1+y)}{1}-\cdots
$$

is of elliptic type, and thus it diverges. Moreover, $\left\{\tilde{t}_{n}\right\}_{n=0}^{\infty}$, where $\tilde{t}_{2 n}=$ $x, \tilde{t}_{2 n+1}=-(1+y)$ for all $n$, is a tail sequence for $K\left(\tilde{a}_{n} / 1\right)$. This tail sequence satisfies $\left(3.2^{\prime}\right)$ since

$$
\prod_{m=j+1}^{j+2} \frac{1+\tilde{t}_{m}}{-\tilde{t}_{m}}=\frac{1+x}{-x} \cdot \frac{-y}{1+y}=e^{-i \theta}, \quad \prod_{m=j+1}^{j+2} \frac{-\tilde{t}_{m}}{1+\tilde{t}_{m}}=e^{i \theta},
$$

and thus, for instance,

$$
\begin{aligned}
\sum_{k=0}^{2 n-1} \prod_{m=2 j}^{2 j-1+k} \frac{1+\tilde{t}_{m}}{-\tilde{t}_{m}} & =\sum_{k=0}^{n-1} \prod_{m=2 j}^{2 j-1+2 k} \frac{1+\tilde{t}_{m}}{-\tilde{t}_{m}}+\sum_{k=0}^{n-1} \prod_{m=2 j}^{2 j+2 k} \frac{1+\tilde{t}_{m}}{-\tilde{t}_{m}} \\
& =\sum_{k=0}^{n-1} e^{i k \theta}+\sum_{k=0}^{n-1} \frac{1+x}{-x} e^{i k \theta}=\frac{1}{-x} \cdot \frac{1-e^{i n \theta}}{1-e^{i \theta}} .
\end{aligned}
$$

Actually, we easily find that $\left(3.2^{\prime}\right)$ holds with

$$
M=\max \left\{\frac{1}{|x|}, \frac{1}{|y|}, \frac{1}{|1+x|}, \frac{1}{|1+y|}, 1\right\} \cdot \frac{2}{\mid 1-e^{i \theta \mid}}<\infty .
$$

From Theorem 3.1A we therefore find that every continued fraction $K\left(a_{n} / 1\right)$ such that $\sum\left|a_{n}-\tilde{a}_{n}\right|<\infty$ diverges.

Final remark. One can relax condition (3.2) and still get results like Theorem 3.1 if one compensates by restricting (3.6).

\section{ACKNOWLEDGMENT}

The author is grateful to Professor W. B. Jones for valuable remarks. It has been brought to the author's attention that [12] may contain results which are related to Theorem 1.1A.

\section{BIBLIOGRAPHY}

1. F. V. Atkinson, Discrete and continuous boundary problems, Academic Press, New York, 1964.

2. J. Gill, Infinite compositions of Möbius transformations, Trans. Amer. Math. Soc. 176 (1973), 479-487.

3. M. E. H. Ismail, D. R. Masson, and E. B. Saff, $A$ minimal solution approach to polynomial asymptotics, Orthogonal Polynomials and their Applications (C. Brezinski, L. Gori, and A. Ronveaux, eds.), J. C. Baltzer AG, Basel, 1991, pp. 299-303.

4. L. Jacobsen, Convergence of limit k-periodic continued fractions $K\left(a_{n} / b_{n}\right)$, and of subsequences of their tails, Proc. London Math. Soc. 51 (1985), 563-576.

5. _ Nearness of continued fractions, Math. Scand. 60 (1987), 129-147. 
6. L. Jacobsen and D. Masson, On the convergence of limit periodic continued fractions $K\left(a_{n} / 1\right)$, where $a_{n} \rightarrow-\frac{1}{4}$. Part 3, Constr. Approx. 6 (1990), 363-374.

7. L. Jacobsen and $\mathrm{H}$. Waadeland, Some useful formulas involving tails of continued fractions, Lecture Notes in Math., vol. 932, Springer-Verlag, Berlin, 1981, pp. 99-105.

8. W. B. Jones and W. J. Thron, Continued fractions. Analytic theory and applications, Encyclopedia of Mathematics and its Applications, vol. 11, Addison-Wesley, Reading, MA, 1980.

9. K. Knopp, Theorie und Anwendung der unendlichen Reihen, Grundlehren Math. Wiss., Bd. 2, Springer-Verlag, Berlin, 1947.

10. L. Lorentzen, A note on separate convergence for continued fractions, J. Comput. Appl. Math. 47 (1993), 123-133.

11. O. Perron, Die Lehre von den Kettenbrüchen, Band 2, Teubner, Stuttgart, 1957.

12. R. Spigler and M. Vianello, Asymptotic representation for a class of orthogonal polynomials via discrete Liouville-Green approximation, J. Approx. Theory (to appear).

13. M. A. Stern, Lehrbuch der algebraischen Analysis, Leipzig, 1860.

14. O. Stolz, Vorlesungen über allgemeine Arithmetik, Teubner, Leipzig, 1886.

15. W. Van Assche, Asymptotics for orthogonal polynomials and three-term recurrences, Orthogonal Polynomials: Theory and Practice (Paul Nevai, ed.), Kluwer, Dordrecht, 1990, pp. 435-462.

16. E. B. Van Vleck, On the convergence of continued fractions with complex elements, Trans. Amer. Math. Soc. 2 (1901), 215-233.

17. H. Waadeland, Tales about tails, Proc. Amer. Math. Soc. 90 (1984), 57-64.

Division of Mathematical Sciences, University of Trondheim - NTH, N-7034 TrondHEIM, NORWAY 